Rev. Elev. Méd. Vét. Pays trop., 1966, 19, I (21-28).

\title{
Essai d'élevage de Glossina tachinoides W. au Laboratoire
}

\author{
por J. GRUVEL et J. BALIS \\ (avec la collaboration technique de Mme J. LANDRY)
}

\begin{abstract}
RÉSUMÉ
Après un rappel des principaux élevages de Glossines en laboratoire, les auteurs exposent les résultats d'un élevage de Glossino tachinordes W. réalisé au laboratoire de Farcha, Fort-Lamy (Tchad). Cette espèce n'avaił été élevée jusqu'à présent qu'une seule fois en 1934 par BUXTON et LEWIS.

L'origine de l'élevage réalisé à Farcha a été constitué por des pupes récoltées près de Fort-Lamy, de février à avril 1963. Les pupes ont été placées en tubes individuels, à $25^{\circ} \mathrm{C}$ et '40 p. $100 \mathrm{~d}$ 'H. R. Les mouches écloses ont été élevées également en tubes individuels, dans les mêmes conditions. Les accouplemenls, réalisés à l'âge de 2 jours, après un repas de sang, ont permis d'obtenir 4 larves, dont deux ont donné des mâles. Les durées de pupaison ont été de 27 et 37 jours.

Les observations faites au cours de cet élevage permettront la mise en route d'un élevage à meilleur rendement. L'infiuence de certaıns facteurs : lumière, degré hygrométrique, source et rythme des repas, doit être précisée.
\end{abstract}

Les études sur la biologie des tsétsés ont été entreprises dès 1895-96 par Sır David BRUCE qui mit en évidence leur mode de reproduction, puis par E. ROUBAUD qui en 1909 définit les deux caractères fondamentaux de leur biologie : I'hématophagie ef la larviparité.

Afin d'élucider de nombreux détails obscurs de la vie ef du rôle pathogène des tsétsés, E. ROUBAUD entreprit à l'Institut Pasteur de Paris, dès décembre 1913, un élevage de Glossina morsitans qu'il entretint jusqu'en 1917. Depuis, de nombreuses études sur la biologie des mouches tsétsés maintenues au laboratorre ont suivi et porté principalement sur :

- Glossina palpalis R. D. : MELLANBY (H.), 1937 ; MELLANBY (H. et K.), 1937 ; RHODAIN (J.) et VAN HOOF (M. T.), 1944 ; GEIGY (R.), 1948; JORDAN (A. M.) et SQUIRE, 1951; NASH (T. A. M.), 1954, 1956 ; NASH (T. A. M.),
$\operatorname{PAGE}($ W.), JORDAN (A. M.) et PETANA (W.), 1958; travaux du W. A. I. T. R. à Kaduna, 1960. - Glossina palpalis martinii Z. : EVENS (F. M.) 1954.

- Glossma fuscipes quanzensis P. . : MAILLOT (L.) 1958.

- Glossina morsitons W. : BROOM (F. C.), 1939 ; WILLET (K. C.), 1953 : NASH (T. A. M.), 1956 ; FOSTER (R.), 1957, 1958 ; PEEL (E.) et CHARDOME, 1958 ; MC DONALD (W. A.), 1960 ; travaux du W. A. I. T. R. à Kaduna, 1960; FORD (J.) et LEGGATE (B. M.), 1961 ; travaux de I'E. A. T.R. O., 1961 : SOUTHON (H. A. W.) et COCKINGS (K. L.), 1963.

Par contre, le maintien de Gl. fachinordes $W$. au laboratoire n'a été signalé qu'une seule fols par BUXTON (P. A.) et LEWIS (D. J.) à Gadau (Nigéria), en 1934. Ces auteurs comparalent les effets des facteurs température et 
humidité dans les conditions naturelles et au laboratoire sur les espèces $G l$. $m$. submorsitons $N$. et $G l$. tachinoides $W$. Dans un document non publié, I'un de nous (*) expose les conditions et les résultats d'un essai d'élevage de Gl. tochinoides $W$. au laboratoire de Farcha, essai réalisé dans des conditions rudimentoires et dans le but de tırer quelques conclusions utıles à une étude ultérieure plus précise.

- Chaque pupe était mise à incuber dans un tube de verre $(50 \times 20)$ fermé par un bouchon de liège, et, dès la naissance, chaque mouche transférée dans un tube à essais bouché par un tampon de coton. Les repas étaient pris quatidiennement sur homme et sur cobaye. La température ambiante se maintenait entre $24^{\circ} \mathrm{C}$ ef $30^{\circ} \mathrm{C}$ avec une humidité d'environ 40 p. 100.

- Les résultats apportés par ce premier essai montraient que :

a) les pupes ne se trouvaient dans la nature que pendant les mois de janvier, février et mars ;

b) le pourcentage d'éclosions fut de l'ordre de 80 p. 100 ; éclosions donnant des mouches viables et des mouches mal développées, conservant l'aspect fripé de leur sortie de pupes et non viables. Le maximum d'éclosions ne fut obtenu qu'à partir de pupes transportées d̀ une température inférieure à $30^{\circ} \mathrm{C}$;

c) la longévité des mouches était très varıable et le plus sauvent imprévisible:

d) I'accouplement semblait se réaliser le mieux 5 ou 6 jours après la naissance ;

e) les durées de gestations observées au laboratoire étaient de 7 à 9 jours, celles de pupaisons d'environ 22 jours.

En tenant compte des remarques fournies par cette étude préalable, une nouvelle tentative d'élevage a été réalisée au laboratoire de Farcha.

\section{Conditions d'élevage, récoltes de pupes et éclosions}

Les pupes nécessaires à l'élevage entrepris en 1963 ont été récoltées dans les gîtes les plus proches du laboratorre (bords du Chari à Riggil, Nord Cameroun), dès le mois de février, époque

(*) BALIS (J.). Rapport annuel I. E. M. V.T. Farcha (1960). de l'année où elles commencent d̀ être abondantes. Aussitôf récoltées et pendant le transport, les pupes ont été placées en petit nombre dans des tubes à essais entourés de coton humide ef maintenus au frais. Ces pupes ont été placées en pièce obscure climatısée à température constante de $25^{\circ} \mathrm{C}$ avec une humidité relative de 40 p. 100 ; chacune étant logée individuellement dans un petit tube fermé par un bouchon de coton.

Chaque mouche éclose fut ensuite introduite dans un tube à essais stérile disposé horizontalement et fermé par un tube à insectes $(50 \times 20)$ laissant aınsi un libre passage à l'air. Quotidiennement la mouche a reçu sur homme ou sur cobaye, un repas de sang, à la suite duquel elle était mise dans un nouveau tube stérile. Les mouches se tenalent à l'embouchure du tube à essais, au niveau de l'entrée d'air.

Les récoltes des pupes ont éfé faites du 12 février au 9 avril 1963 à des intervalles irrégulıers (*). Aucune pupe n'a été trouvée en dehors de la période comprise entre le 5 février et le 9 avril. Au total 266 pupes ont été mises en élevage: 204 ont donné naissance à des Glossines, 7 on produit des Thyridanthrax argentifrons Austen et 55 n'ont pas éclos.

Parmi les 204 tochinordes à terme, 41 seulement (15 mâles et 26 femelles) étaient parfaitement constituées et 163 restèrent sous l'aspect dit "araignée» (selon l'expression de GEIGY, 1948) caractérisé par les ailes non déplissées e† le proboscis mou. Ces dernières moururent rapidement (tableau 1 et graphique 1). (Au terme «araignée » qui nous paraît impropre, nous substituerons celui de mouches «non évoluées ».)

La durée totale de la pupaison est difficile à préciser puisqu'on ignore l'âge des pupes prélevées dans la nature. Au laboratoire des délass de 1 à 32 jours ont été observés entre la récolte de la pupe et l'éclosion de la mouche. Une durée d'au moins 32 jours apparaît donc nécessaire à l'élaboration de la mouche dans la pupe. Ces résultats sont à comparer avec les durées de pupaisons effectuées totalement au laboratoire et qui furent de 37 et 27 jours.

(*) Nous avons pu observer dès le 5 février 1963 (vers 15 heures) dans un gîte, la ponte d'une larve dont la pupe n'a pas été élevée. 


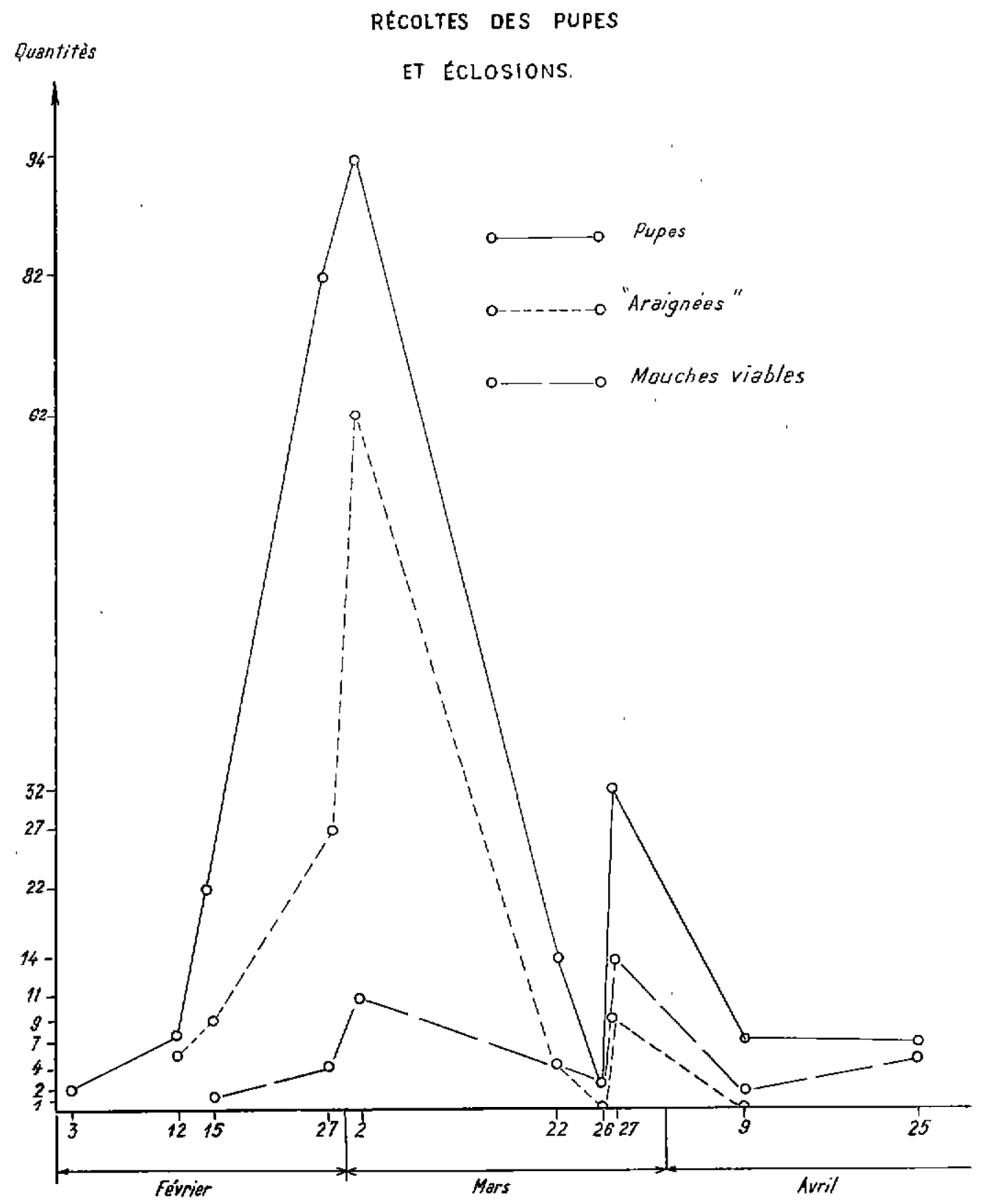

GRAPHIQUE $N: 1$ 
TABIOAN NO I

RÉcolte des pupes et éciosions

(Glossina tachanoides W.)

\begin{tabular}{|c|c|c|c|c|c|c|c|c|c|c|c|c|c|c|}
\hline \multirow{3}{*}{$\frac{\text { Mois }}{\text { Jours }}$} & & & & \multicolumn{9}{|c|}{ Hoe en élevage } & \multirow{3}{*}{$\frac{\text { Mai }}{2}$} & \multirow{3}{*}{ Totaix } \\
\hline & \multicolumn{3}{|c|}{ Janyer } & \multicolumn{3}{|c|}{ Févries } & \multicolumn{4}{|c|}{ Mars } & \multicolumn{2}{|c|}{ Avril } & & \\
\hline & 23 & 30 & 3 & 12 & 15 & 27 & 2 & 22 & 26 & 27 & 9 & 25 & & \\
\hline Nombre de pupea & 0 & 0 & 1 & 7 & 22 & 82 & 94 & 14 & 2 & 32 & 7 & 6 & 0 & 266 \\
\hline $\begin{array}{l}\text { Eclosions adultes } \\
\text { viables }\end{array}$ & & & & & 1 & 4 & 11 & 4 & 2 & 14 & 1 & 4 & & 41 \\
\hline $\begin{array}{l}\text { Eclosions } \\
\text { "Arazgnées" }\end{array}$ & & & & 6 & 9 & 27 & 68 & 4 & & 9 & & & & 163 \\
\hline 'Thyridanthrax & & & & & & & & 3 & & & 3 & 1 & & 7 \\
\hline non éclos & & & & 1 & 12 & 11 & 15 & 3 & & 9 & 3 & 1 & & 55 \\
\hline $\begin{array}{l}\text { Pourcentage } \\
\text { adultes vables }\end{array}$ & & & & & 4,5 & 4,8 & 11,7 & 28,6 & 100 & 49,9 & 14,3 & 66 & & 15 \\
\hline $\begin{array}{l}\text { Pourcentage } \\
\text { "Araignées" }\end{array}$ & & & & & 40 & 81 & 72 & 29 & & 28 & & & & 61 \\
\hline
\end{tabular}

D'autre part, en 1960, BALIS nota 22 jours pour une seule pupaison observée en totalité à une température supérieure à $25^{\circ} \mathrm{C}$. Ces variations dans les durées de pupaisons peuvent s'expliquer par les différences de température ; une élévation de température (dans les limites compatibles avec la vie) accélérant les métabolismes.

Si l'on adopte le chiffre de 32 jours comme durée moyenne de la pupaison on peut déduire approximativement d'après les dates d'éclosion que les pontes ont été effectuées dans une période située entre le 1 er février et le 5 avril, avec un maximum dans la 2 e quinzaine de février (cf. graphique 2).

\section{Repas et digestion}

Le premier repas était pris une dizaine d'heures après la naissance; avant ce délai les mouches ne peuvent piquer en raison du manque de rigidité du proboscis. La durée du repas était variable selon la voracité des mouches: cependant, leur précipitation sur la source de nourriture était sans relation avec l'importance du repas. L'expérience a montré qu'un jour de jeûne par semaine était parfaitement supporté.
Rappelons que le changement de tube d'élevage a toujours été quotidien et effectué aussitôt après le repas.

La digestion normale était illustrée par une émission quotidienne d'excréments se présentant sous la forme de masses noires disposées régulièrement à la partie inférieure du tube.

Cependant la digestion peut subir des modifications qui permettent d'apprécier l'évolution de l'élevage. Ces modifications étant produites, soit par perte de l'appétit aboutissant à une deshydratation et à la mort, soit par excès d'appétit entraînant purement et simplement l'éclatement de l'abdomen. Ces observations faites fréquemment nous ont conduit à interrompre le repas des mouches se montrant trop voraces. Une autre cause de mort, inconnue, mais d̀ symptômes digestifs, se traduisait par un gonflement de l'abdomen accompagné d'une émission d'excréments teintés du sang absorbé ; la mort suivait rapidement l'apparition de ces signes.

\section{Reproduction}

Six accouplements furent obtenus au laboratoire. La femelle était introduite dans le tube d'un mâle et l'union avait lieu immédiatement 


\section{DURÉES DES PUPAISONS ET DES SURVIES}

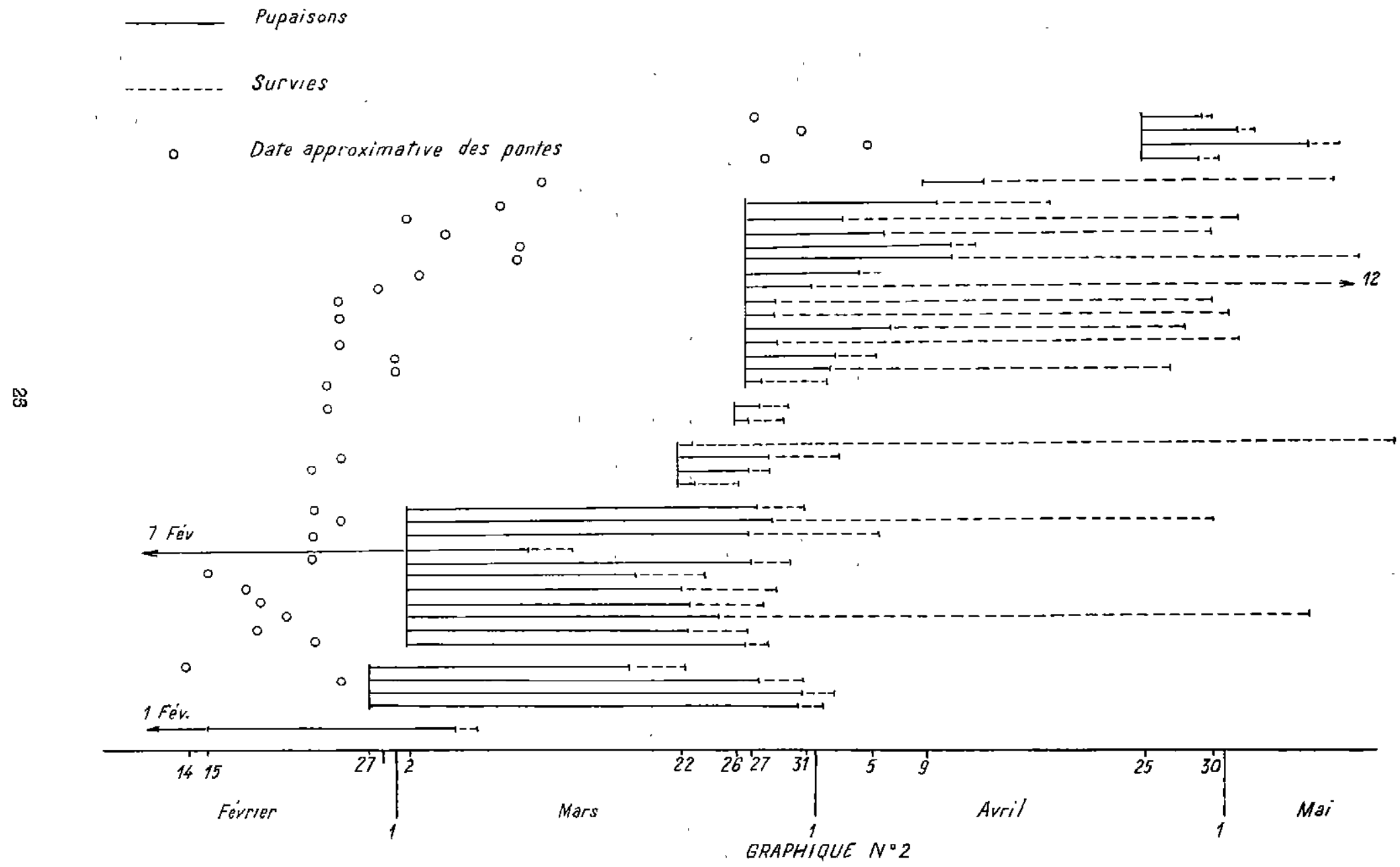


après. Seules les mouches âgées de 2 jours et ayant pris au moins un repas de sang se sant accouplées. Sur six tentatives d'accouplements, une seule ne s'est pas réalisée. Une femelie seulement a donné naissance à 4 larves pondues respectivement $16,27,39$ et 57 jours après la fécondation, ce qui correspond à des durées de gestation de $16,11,12$ et 18 jours. Les gestations plus courtes de 7 à 9 jours, observées en 1960 par BALIS, s'expliquent là encore par la température plus élevée du local d'élevage. A partir de ces 4 larves, 4 pupes se sont formées et 2 seulement ont donné issue à des mâles qui n'ont pas vécu. Ces durées de pupaisons ont été de 37 et 27 jours. La mère a vécu au total 73 jours (tablequ 2).

\section{Cos des mouches «non évoluées》}

D'une manıère générale les mouches prennent leur aspect définitif dans les premières heures qui suivent la naissance. Cet aspect définitif est précisé par la rigidité du proboscis et l'étalement complet des ailes.

Cependant, quelques mouches n'ont déplissé leurs ailes qu'au bout de 1 et 2 jours, une seule a survécu 8 jours sans repas et ce n'est qu'après ce délai inhabituel que les ailes sont devenues normales et que le premier repas a été pris.

Un grand nombre de mouches ne subit pas cette évolution et se maintient à ce stade au cours duquel aucune d'elles n'est capable de se nourrir, sa trompe se pliant sans pénétrer le tégument.

TABLEAU NO II

Accouplements

\begin{tabular}{|c|c|c|c|c|c|c|c|c|c|c|c|}
\hline \multicolumn{2}{|c|}{ H. $\times \mathrm{F}$. } & \multicolumn{2}{|c|}{ Accouplement } & \multirow[t]{2}{*}{$\begin{array}{r}\text { 1ère } \\
\text { ponte }\end{array}$} & \multirow[t]{2}{*}{$\begin{array}{c}\text { 1̇̉re } \\
\text { naissance }\end{array}$} & \multirow[t]{2}{*}{$\begin{array}{l}\text { 2ème } \\
\text { ponte }\end{array}$} & \multirow[t]{2}{*}{$\begin{array}{c}\text { 2ème } \\
\text { naissance }\end{array}$} & \multirow[t]{2}{*}{$\begin{array}{l}\text { Sènio } \\
\text { ponte }\end{array}$} & \multirow[t]{2}{*}{$\begin{array}{c}\text { 3ène } \\
\text { naissance }\end{array}$} & \multirow[t]{2}{*}{$\begin{array}{l}\text { 4ème } \\
\text { ponte }\end{array}$} & \multirow[t]{2}{*}{$\begin{array}{c}\text { 4ème } \\
\text { naissance }\end{array}$} \\
\hline$n^{0} \quad 48 x$ & 148 & + & 22.3 .63 & & & & & & & & \\
\hline $116 x$ & 217 & + & 25.3 .63 & & & & & & & & \\
\hline $48 x$ & 158 & + & 21.3 .63 & & & & & & & & \\
\hline $116 x$ & 217 & + & 25.3 .63 & & & & & & & & \\
\hline $170 x$ & 226 & - & 29.3 .63 & & & & & & & & \\
\hline $178:$ & 238 & + & 8.4 .63 & 24.4 .63 & 31.5 .63 & 5.5 .63 & - & 17.5 .63 & 13.6 .63 & 3.6 .63 & - \\
\hline
\end{tabular}

L'origine de cette anomalie n'est pas déterminée : elle ne semble pas due à des conditions d'élevage particulıères puisque dans les mêmes circonstances on obtient également des mouches normales. Il est possible qu'elle existe dans la nature, mais c'est un fail difficile à préciser car ces mouches non évoluées sont sans défense et doivent mourir rapidement d'inanition ou victimes de prédateurs.

\section{Cas des mouches prises dans la noture}

Quatre mouches (2 mâles et 2 femelles) capturées dans la nature ont pu être gardées vivantes au laboratoire; elles ont vécu 16, 3, 4 et 4 jours et ont pris régulièrement leur repas de sang.
Elles se sont maintenues en parfaite santé pendant toute leur vie et sont mortes sans cause apparente.

Ce fait est intéressant à signaler car jusqu'à présent aucune survie n'avait pu être obtenue à partir de tochinordes capturées dans leur milieu (tableau 3).

TABLEAU NO III

Survie des mouches sauvages

\begin{tabular}{|c|c|c|c|c|}
\hline Date de capture & \multicolumn{4}{|c|}{25 avril 1963} \\
\hline Survie (jours) & 16 & 3 & 3 & 4 \\
\hline Sexe & F. & N. & F. & M. \\
\hline
\end{tabular}




\section{CONCLUSIONS}

Ces observations permettent de tirer desconclusions favorables à la mise en route d'un élevage dà meilleur rendement. L'influence de certains facteurs reste à préciser, notamment la lumière, le degré hygrométrique, les sources de nourriture et le rythme des repas.

Cette forme d'élevage, de type individuel, 'présente de nombreux inconvénients puisqu'elle demande des soins constonts, un personnel entièrement attaché à son entretıen et ne permet de garder qu'une faible quantité de mouches. Par contre, il rend possible l'étude du compor- tement de chaque mouche individuellement.

C'est le seul mode d'élevage qui nous ait donné quelques résultats. Toutes les tentatives pour faire vivre Gl. tochinordes en cages Roubaud ou en grandes cages, que ce solt dans leurs propres gîtes ou qu laboratoire, se sont soldées par des échecs; les mouches mourant dans les six heures qui suivalent la mise en cage.

Compte tenu de ces premières remarques, un autre essai d'élevage est à l'étude.

Institut d'Elevage et de Médecine

Vétérinaire des Pays tropicaux

Laborotore de Forcho (Fort-Lamy. Tchad).

\section{SUMMARY}

Attempt to breed Glossina tachinoides $W$ in a laboratory

After recalling the principal laboratory glossince breedings, the authors describe the results of a Glossina tachinoides $W$. breeding in the Fort-Lamy (Chad) laboratory. This species has been kept in such conditions only once before, in 1934 by BUXTON and LEWIS.

The origin of the breeding in Farcha comes from pupae collected around Fort-Lamy between february and april 1963. The pupae have been placed in individual tubes at $25 \mathrm{C}$ degrees of temperature and $40 \mathrm{p.} 100$ of moisture. The hatched flies are equally kept in individual tubes in the same conditions.

Mating, realised at the age of 2 days, after a meal on blood, gave birth to 4 larvae two of which were males. Pupae maturing took 27 and 37 days.

Observations made during this experiment will help establishing a more fruitful breeding. The influence of some factors : light, moisture, nature and rythm of meals must be made mare precise.

\section{RESUMEN}

\section{Ensayo de cria de Glossina lachinoides $W$ en el laboratorio}

Después de una reseña de las principales crias de glosinas en el laboratorio, los autores exponen los resultados de una cria de Glossina tachinoides W. en el laboratorio de Fort-Lamy (Chad). Hasta ahora BUXTON y LEWIS habian criado esta especie solo una vez en 1934.

La cria fué realızada en Farcha a partır de pupas recogidas cerca de FortLamy, desde febrero hasta abril de 1963. Se pusieron las pupas en tubos individuales con una temperatura de $25^{\circ} \mathrm{C}$ y 40 por 100 de humedad. Se criaron también las moscas nacidas en tubos individuales, en las mismas condiciones. Los acoplamientos, a 2 días de edad, después de una comida de sangre, permitieron obtener 4 larvas, entre las cuales dos fueron machos. El tiempo de la pupación fue de 27 y 37 días.

Se utilizará estas observaciones para otra cria con mejor termino. Es necesario notar la influencia de ciertos factores : luz, grado higrométrico, origen y ritmo de las comidas. 


\section{BIBLIOGRAPHIE}

BROOM (F. C.). - The maintenance of Glossina morsitans in England for experimental work. Trans. Roy. Soc. Trop. Med. ef Hyg., 1939, 32 (5).

BUXTON (P. A.) et LEWIS (D. J.). - Climate and Tsetse flies : laboratory studies upon GI. submorsitans and tachinoides. Philos. Trans., 1934 (B) : 224, 175-240.

Mc DONALD (W. A.). - The laboratory rearing of Gl. m. submorsitans in Northern Nigeria. Int. Sci. Comm. for Trypano Res.

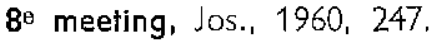

East African Trypanosomiasis Research Organisation. Annual Report, 1961, 31.

EVENS (F. M.). - Recherches sur l'élevage et la biologie de Gl. palpalis martinii. Bruxelles Inst. R. Sci Nat., 1954.

FORD (J.) et LEGGATE (B. M.). - The geographical and climatic distribution of trypanosome infection rates in Gl. morsifans group of tsetse. Trans. Roy. Soc. Trop. Med. Hyg., 1961, $55: 383$.

FOSTER (R.). - Colonies de mouches tsétsés au laboratoire. Parasitology, 1957, $47: 361-374$.

FOSTER (R.). - Colonies de mouches tséłsés Gl. morsitans en laboratoire. CCTA - B. P. I. T. - Trypanosomiase. Public no 217/T., 1958.

FOSTER (R.). - Some observation on the breeding of Gl. morsitans in The laboratory. Int. Sci Comm, for Trypano. Res., 7e meeting Brussel, 1958 : 351.

GEIGY (R.). - Elevage de Glossina palpolis. Acta tropica., 1948, 5 (3) : 201-217.

JORDAN (A. M.). - The mating behaviour of females of $G$. palpolis in captivity. Bull. Ent. Res., 1951, 49, 3.

MAILLOT (L.). - Elevage de Gl. fuscipes quanzensis P. à Brazzaville. Bull. I. E. C. no 15-16, $1958: 85-90$

MELLANBY $(H$.$) .- Experimental work on repro-$ duction in the tsetse fly Glossina palpalis. Parasitology., 1,937, $29: 131-141$.

MELLANBY ( $H$. et K.). - Rearing tsetse flies in captivity. Proc, Roy. Entomol. Soc. London (A)., 1937, 12:1-3.

NASH (T. A. M.). - Laboratory investigations. West African Institute for Trypanosomiasis
Research, Kaduna. Annual Report., 1954 : 30-31.

NASH (T. A. M.). - Laboratory investigations : a) the mating behaviour of female Gl. palpalis in captivity ;

b) Laboratory rearing of Gl. palpalis ;

c) laboratory rearing of Gl. morsifons. West. Afr. Inst. for Trypano. Res., Kaduna. Annual Report., $1956: 29-33$.

NASH (T. A. M.). - Progress and problems in the establishment and maintenance of Laboratory colonies of tsetse flies. Bull. Org. Mond. Santé, 1963, 28 (5) : 831-836.

NASH (T.A.M.), PAGE (W.A.), JORDAN (A.M.), PETANA (W.). - The rearing of GI. polpalis in the laboratory for experimental work. Sc, Comm. for Trypano. Res., 1958 : 343, 7 e meeting, Brussel.

PEEL (E.) et CHARDOME (M.). - Observations sur les élevages de Gl. morsitans au Laboratoire. Ann. Soc. Belg. Med. Trop., 1958, 38 (5) : 961-4.

POTTS (W. H.). - Laboratory investigations. Tsetse Research Report 1935-38; Tanganyika Territory; Dar-es-Salam., 1940:48-53. RODHAIN (J.) et VAN HOOF (M. T.). - Au sujet d'un élevage de Glossina palpalis en Europe ef de quelques essais d'évolution chez cette Glossine des Trypanosoma lewisi et cruzi. Ann. Soc. Belge Med. Trop., 1944, 24 : 54-57. ROUBAUD (E.). - Histoire d'un élevage de Gl. morsitans à l'Institut Pasteur de Paris. Bull. Soc. Patho. Exot., 1917, I (X) : 629-640. SOUTHON (H. A. W.) et COCKINGS (K. L.). Laboratory maintenance of Glossina morsitans. East Afr. Tryp. Res. Organisation Report., 1963: 31.

SQUIRE ( $F$. A.). - Observations on mating scars in Glossina palpalis. Bull. Ent. Res., 1951, $42: 3$.

VAN DER PLANK (F. L.). - Experiments in - cross-breeding tsetse flies species. Ann. Trop. Med. et Paras, 1948, 42 (2) : 131-52.

WEST AFRICAN INSTITUTE for Trypanosomiasis Research, Kaduna. The laboratory rearing of Gl. palpalis. Annual Report, p. 49.

WILLET (K. C.). - The laboratory maintenance of Glossina, 1. Parasitology., 1953, $43: 110$. 